\title{
Intermediación laboral y mercados de trabajo en agriculturas reestructuradas: el caso del Valle de Uco, Mendoza, Argentina
}

\author{
Germán Quaranta* \\ Francisco Fabio **
}

Resumen: Por tradición, la intermediación laboral en la agricultura ha facilitado el reclutamiento, la movilización y el control de la fuerza de trabajo. En la actualidad, en contextos de agriculturas reestructuradas social y productivamente, ésta modifica su perfil y características; la diversidad de sus modalidades y funciones permite a las empresas utilizar estrategias muy flexibles de contratación, a la vez que posibilita formas diferentes de registro de los trabajadores. Las producciones agrícolas del Valle de Uco, en la provincia de Mendoza, Argentina, constituyen un caso con gran potencial para avanzar en la conceptualización de la intermediación laboral en este tipo de escenarios. La heterogeneidad de formas, funciones y actores conforman un panorama a partir del cual es posible extraer conclusiones sobre su naturaleza en las condiciones señaladas.

Palabras clave: intermediación laboral, flexibilidad laboral, movilización, agricultura reestructurada.

* Investigador adjunto del Consejo Nacional de Investigaciones Científicas y Técnicas (CONICET), área empleo y desarrollo rural, Centro de Estudios e Investigaciones Laborales-Programa de Investigaciones Económicas sobre Tecnología, Trabajo y Empleo (CEIL-PIETTE/CONICET). Saavedra 15, piso 4, Ciudad de Buenos Aires ACA1083, Argentina. Teléfono: (54) 114952 7440. Correo electrónico: gquaranta@ceil-piette.gov.ar

** Becario doctoral tipo II del CEIL-PIETTE/CONICET. Correo electrónico: ffabio@ceil-piette. gov.ar 
Abstract: This article addresses the characteristics of labor intermediation in the context of restructured corporate agriculture, focusing on the role of the phenomenon in shaping labor markets. Traditionally, labor intermediation in agriculture facilitated labor force recruitment, mobilization and control, primarily through coercive means. Nowadays, labor intermediation in agricultural contexts which have been restructured socially and productively has changed its profile and characteristics. The diversity of its modalities and functions allows companies to use highly flexible strategies for labor recruitment, while allowing different forms of worker registration. Agricultural production in the Uco Valley, in the province of Mendoza, Argentina, is a case in point with great potential to improve the conceptualization of labor intermediation in this type of scenario. The diversity of functions and the variety of actors involved constitute a scenario from which conclusions can be drawn about the nature of the phenomenon under the conditions mentioned above.

Key words: labor intermediation, labor flexibility, mobilization, restructured agriculture.

\section{Introducción}

Los mercados de trabajo agrícola experimentan transformaciones profundas en el contexto de la reestructuración social y productiva del agro, en las condiciones actuales del desarrollo capitalista. En este marco, las estrategias empresariales persiguen - en diferente medida y según el caso- modalidades cuantitativas y cualitativas de flexibilidad laboral en busca de sostener o profundizar la acumulación de capital (Lara Flores 1998; Neiman y Quaranta 2000; Radonich et al. 2007). En los escenarios nuevos, la intermediación laboral modifica sus características y significados, y plantea otras interrogantes sobre sus modalidades, funciones y el papel que cum- 
ple en la estructuración y el funcionamiento de los mercados de trabajo.

En este artículo se abordan las características adquiridas por la intermediación laboral en la agricultura empresarial, que pasa por procesos de reestructuración profundos, y su papel en la conformación de los mercados de trabajo. La metodología empleada combina datos cuantitativos y cualitativos; los primeros permiten, a partir de procesamientos especiales del Censo nacional agropecuario, CNA (Instituto Nacional de Estadísticas y Censos, INDEC 2002), analizar el uso de la intermediación laboral según la tarea realizada y el tamaño de los establecimientos involucrados. Con respecto a los cualitativos, a través de entrevistas no estructuradas se abordan las peculiaridades de la intermediación laboral según sus modalidades y funciones, así como sus efectos sobre las condiciones presentes en los mercados de mano de obra.

Por tradición, la intermediación laboral en la agricultura ha facilitado el reclutamiento, movilización y control —sobre todo a través de medios coercitivos- de la fuerza de trabajo. En la actualidad, en contextos de agriculturas reestructuradas social y productivamente, ésta modifica su perfil y características; la diversidad de sus modalidades y funciones permite a las empresas adoptar estrategias de contratación muy flexibles, a la vez que posibilita, en algunos casos, formas diversas de registro de los trabajadores.

\section{La intermediación laboral en contextos diferentes del desarrollo del capitalismo en la agricultura}

El estudio de la intermediación laboral y su papel en la conformación, las características y la dinámica de los mercados de trabajo agrario posee una larga tradición académica, vinculada a situaciones y momentos distintos del desarrollo del capitalismo en la agricultura. Hubo elementos clave en la conformación y difusión de dicho fenómeno, como la demanda de grandes volúmenes de personal, requerido en momentos puntuales y por periodos determinados; el deseo de los establecimientos de operar en condiciones de sobreoferta de trabajo, de forma tal que debilita la capacidad de 
acción y de disputa de las condiciones de ocupación de los empleados; la necesidad de articular una demanda laboral como la indicada y una oferta de mano de obra, en muchas ocasiones distantes, entre otros (Fisher 1964).

Las instancias de intermediación laboral constituyen para el capitalista una forma eficaz de resolver el problema clásico de la estacionalidad de los requerimientos de trabajo en la agricultura donde: "Siempre hay demasiados obreros agrícolas para las necesidades medias y demasiado pocos para las necesidades excepcionales o temporales de la misma" (Marx 1975, 867). La modalidad difundida para solucionar esta situación al este de Inglaterra, según relata Marx, ${ }^{1}$ fue el sistema de cuadrillas (gang-system) que permitía a los agricultores capitalistas movilizar a los jornaleros necesarios en los periodos específicos de requerimiento, sin necesidad de preocuparse por su reclutamiento como tampoco, en tanto productor individual, por la disponibilidad y reproducción. Las cuadrillas, al desplazarse de una explotación a otra, buscaban mantener a sus miembros ocupados el mayor tiempo posible, y así garantizaban esa disponibilidad y, en condiciones paupérrimas, su reproducción.

La intermediación laboral, entendida como instrumento de articulación de la oferta y la demanda de trabajo a partir de la acción de un tercer actor social, asumió modalidades distintas y cumplió funciones variadas en las etapas y momentos del avance del capitalismo agrario. Ésta desempeñó un rol destacado en la conformación de los mercados asalariados de trabajo en América Latina y Argentina. En un primer momento se asoció a elementos de coerción y control de la mano de obra, a formas compulsivas de reclutamiento y migración y a mecanismos de endeudamiento, destinados a forzar la asalarización de segmentos determinados de la población. Así, la conformación de mercados temporales de trabajo abastecidos por migrantes, sobre todo campesinos semiproletarizados, movilizados por estos enganchadores, a través de dispositivos de coerción o mecanismos de endeudamiento, constituían los componentes principales del fenómeno.

\footnotetext{
${ }^{1}$ Ver el acápite "El proletariado agrícola británico", en el capítulo xxIII "La Ley General de Acumulación Capitalista” del libro primero de El capital.
} 
En el norte de Argentina, por ejemplo, es reconocida la importancia de estos elementos en la constitución de los mercados de trabajo asociados a la producción azucarera del valle del río San Francisco, en las provincias de Salta y Jujuy. Los ingenios, con el propósito de asegurar la cantidad enorme de brazos necesarios para la zafra, arrendaron o adquirieron haciendas en las tierras altas de Salta y Jujuy, que se encontraban en una situación crítica debido a su marginalidad en la dinámica del nuevo sistema económico ligado al litoral pampeano. Los campesinos de estas haciendas ahora debían pagar la renta de la tierra que ocupaban prestando servicios en los campos de los ingenios. Los administradores se desempeñaban como enganchadores encargándose de asegurar la prestación laboral necesaria para el desenvolvimiento de la actividad (Rutledge 1987; Reborati 1974). La obligación de prestar servicios pronto se transformó en necesidad, con el avance de la penetración capitalista y la desarticulación de la economía campesina. Se conforma un espacio de "enclave" de producción capitalista que funciona asociado a "mercados de trabajo satelizados" a partir de los mecanismos de "enganchamiento", así se caracterizaba tempranamente como precario a un trabajador temporal (Bisio y Forni 1976).

En Argentina, durante el periodo de industria sustitutiva de importaciones, ${ }^{2}$ la expansión de los cultivos regionales de cosecha manual (localizados en provincias fuera de la zona pampeana), realizados por empresas medianas y grandes que enfrentaban déficits de oferta de mano de obra local, constituyeron un centro de atracción para los residentes en áreas con excedentes relativos, con respecto a sus requerimientos y capacidades productivas (por lo general, zonas minifundistas o de economía campesina). La presencia de contratistas era común en torno al sistema de migraciones laborales vigente en el noroeste. "El contratista es un personaje clave en la comprensión del funcionamiento de las migraciones estacionales, $y$, como se puede ver, es el verdadero nexo de articulación entre oferta y demanda de trabajadores, y posee la capacidad de regular los flu-

\footnotetext{
${ }^{2}$ En la Argentina este lapso se extendió desde la crisis de la década de 1930 hasta mediados de la de 1970 .
} 
jos de migrantes según cuál sea el comportamiento de la demanda" (Reborati y Sabalain 1981, 29).

Así, se observa cómo la centralidad de los sistemas de intermediación laboral en la agricultura se relaciona con el avance de la explotación capitalista de cultivos con uso intensivo de mano de obra, cuyos problemas para afrontar una escasez relativa de fuerza de trabajo — sobre todo en los momentos de cosecha - requiere asegurar fuentes de aprovisionamiento y generar mecanismos de movilización de trabajadores. A su vez, dicha mediatización de la relación económica contractual permite diluir la responsabilidad legal del empleador, alentar la competencia y la atomización de los empleados, delegar en el intermediario las labores de contención de conflictos abiertos o potenciales y, en suma, favorecer el desdibujamiento de la confrontación de clase entre capital y trabajo.

La intermediación laboral complica su conformación cuando las relaciones sociales establecidas adquieren un carácter interétnico. En estas condiciones, hay capitales culturales indispensables y la intermediación cultural constituye un aspecto clave. Así, el intermediario opera como intérprete y traductor, que facilita la articulación de grupos sociales, donde las diferencias étnicas se yuxtaponen a las condiciones de clase en los procesos de estructuración del orden social (Sánchez Saldaña 2006).

Los procesos de reestructuración social y productiva de la agricultura revitalizaron a la intermediación laboral. Estudios recientes le otorgan significados nuevos en contextos socioeconómicos propios de sociedades del capitalismo avanzado o en producciones modernizadas por completo. La intermediación laboral se constituye a partir de la presencia de agentes que desempeñan una gama variada de funciones. Por ejemplo, pequeños prestadores de servicios de carácter informal o empresas pueden encargarse del reclutamiento, transporte, supervisión, financiamiento y - en caso de ser necesario- del alojamiento de los trabajadores (Marañón 2000). La labor de dichos agentes se asocia con estrategias empresariales de gestión de mano de obra, que flexibilizan su contratación y uso, lo que asegura su movilidad elevada (Pedreño Cánovas 1999; Castellanos Ortega y Pedreño Cánovas 2001). 
En el marco de agriculturas empresariales y modernizadas; el resurgimiento de modalidades de intermediación laboral es un componente que flexibiliza la contratación y organización laboral para sostener sus procesos de acumulación. "En un sentido importante, dado la creciente competencia global entre las empresas agroindustriales, el sistema de intermediación en Inglaterra no es meramente una anomalía histórica (='prácticas medievales de trabajo') sino un aspecto de los procesos de acumulación, una condición sine qua non de la reproducción capitalista en zonas rurales" (Brass 2004, 323) (traducción propia). A esto se suma que en algunos casos la intermediación laboral se asocia de forma creciente con la contratación de trabajadores locales, en lugar de limitarse a la movilización de los provenientes de regiones de menor desarrollo, consideradas por la literatura como "zonas de expulsión”.

De esta manera, la intermediación laboral se redefine en el contexto de los procesos de reestructuración social y productiva de las actividades agrícolas. Las características que ésta asume son el resultado, en gran medida, de respuestas locales a condiciones globales y estructurales que se traducen en su heterogeneidad creciente. El Valle de Uco tiene un gran potencial para avanzar en la conceptualización de la intermediación laboral en las producciones agrícolas, que pasan por procesos de reestructuración social y productiva. La diversidad de formas, funciones y actores involucrados constituyen un escenario a partir del cual es posible extraer conclusiones sobre la naturaleza del fenómeno en las condiciones señaladas.

\section{Algunas consideraciones generales sobre los procesos de reestructuración y la intermediación laboral en el Valle de Uco}

El Valle de Uco - integrado por los departamentos de San Carlos, Tupungato y Tunuyan - forma parte de la provincia de Mendoza, localizada en el centro-oeste de la Argentina, en la región de Cuyo, y limita hacia el oeste con la cordillera de Los Andes. Esta ubicación le proporciona cualidades climáticas, topográficas y de disponibi- 


\section{Figura 1}

Valle de Uco, provincia de Mendoza, Argentina

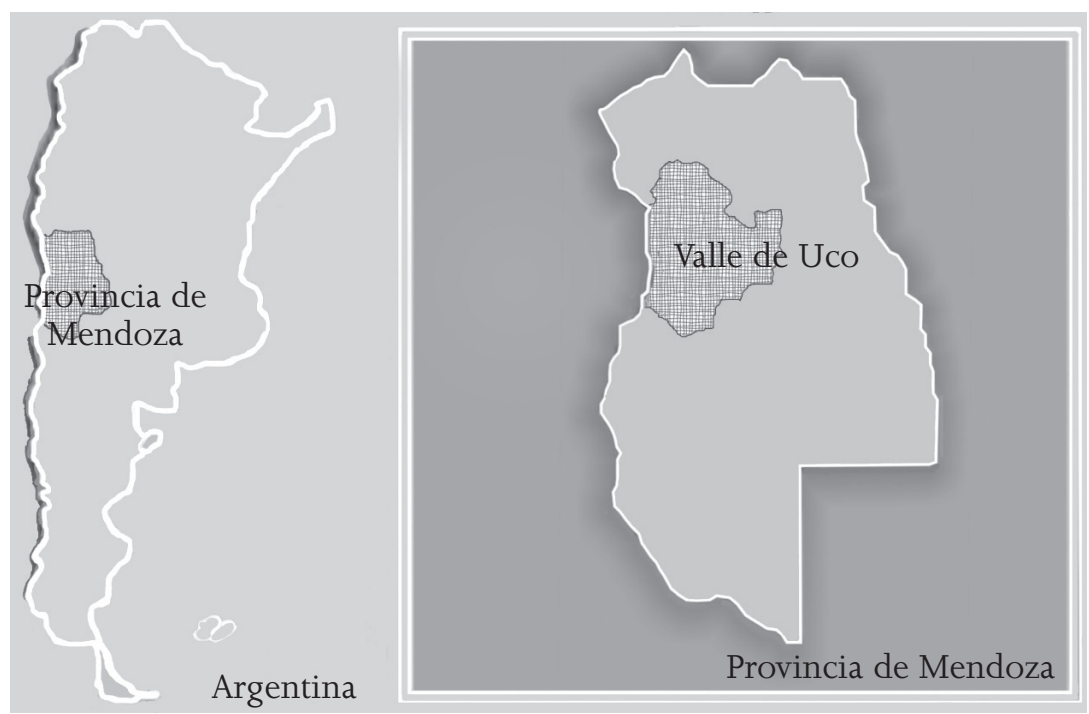

lidad de agua para riego que la hacen particularmente apta para la producción agrícola intensiva.

De manera similar a las restantes zonas irrigadas de la provincia, el Valle de Uco basó su crecimiento económico en producción agrícola y agroindustrial, en especial en la vitivinicultura. La importancia de la agricultura intensiva se manifiesta de manera clara en la estructura ocupacional de la región, ya que 41.1 por ciento de la población labora en la actividad primaria, de la cual 82.3 por ciento corresponde a la categoría de obrero o empleado, cifra que implica al menos a 10600 asalariados, según el Censo nacional de población, hogares y vivienda (INEDC 2001).

En las últimas décadas, la agricultura provincial —en general-y la del valle - en particular-constituyen un escenario socioproductivo nuevo, en este proceso destaca un replanteo de las condiciones que dieron forma al modelo productivista que había estructurado hasta ahora la vitivinicultura. La tendencia principal se resume en 
el paso de un sistema de producción basado en la cantidad, a otro con base en la calidad y orientado hacia la elaboración de bienes diferenciados que satisfagan las necesidades de segmentos específicos del mercado de consumo. De este modo, el concepto de calidad se conformó en un objetivo central de la actividad, y comenzó a estructurar las relaciones que se establecen entre los actores vinculados a ella (Neiman 2003).

Como parte de este proceso se consolida la tradición de la provincia de Mendoza, especializada en cultivo de uva para vinificación, y en este marco el Valle de Uco ha sido el espacio preferido por los emprendimientos nuevos que lideraron la tendencia. El avance del área cultivada con vid por parte de estos emprendimientos provocó que entre 1991 y 2003 en la superficie de esta región se duplicaran los viñedos, ${ }^{3}$ de 7779 a 15250 hectáreas, mientras que el comportamiento de las otras zonas se mantuvo sin grandes variaciones o disminuyó su superficie con viñedos. En este marco de modernización, el avance de la estructura agraria expresa una tendencia hacia la desaparición de unidades, por un lado y, por otro a la concentración de la tierra en unidades de mayor tamaño relativo, que consolida un modelo productivo concentrado en grandes establecimientos. ${ }^{4}$

Por último, como manifestación de los procesos y elementos señalados en fecha reciente, es posible argumentar que la reconversión productiva del sector vitícola mendocino ha generado una redefinición importante de los rasgos con los que se estructura la transitoriedad del ciclo laboral. La mayor importancia relativa de las tareas vinculadas a la calidad de la producción vitivinícola (podas, raleos, desbrotes y controles de riego) agrega mayor equilibrio al ciclo anterior, centrado en la demanda para la cosecha y, de esta manera, repercute profundamente en las estrategias laborales de los empresarios y trabajadores de la región (Neiman et al. 2001; Fabio 2006).

\footnotetext{
${ }^{3}$ Datos del proyecto "Transformaciones productivas y demanda de mano de obra en el agro argentino, 1988-2002”, Instituto Nacional de Tecnología Agropecuaria, INTA-CEIL.

${ }^{4}$ La totalidad de las unidades, como de la superficie que disminuye lo hace en los establecimientos de hasta 25 hectáreas de área total.
} 
Sumado a esto, es importante destacar que si bien la producción vitícola se transformó con rapidez en la actividad agrícola y agroindustrial emblemática de la provincia, desde temprano coexistió con emprendimientos empresariales sobresalientes, vinculados a la fruticultura y horticultura. En ese sentido, en el Valle de Uco las cosechas de durazno, ciruela, manzana, pera, cebolla, tomate, ajo, calabaza y orégano son significativas, y están entre las principales que repercuten en la conformación de una estructura productiva mucho más equilibrada y diversificada que en el resto de la provincia (Bocco et al. 1999; Fabio 2006). ${ }^{5}$ Dichos cultivos se estructuran en la región a partir de unidades empresariales de escala distinta, y se distinguen por el uso intensivo de mano de obra y por conformar calendarios ocupacionales que, vinculados con los procesos productivos y socio-laborales recientes, sobresalen por una mayor transitoriedad en la demanda de empleo.

A su vez, esta modificación en las características del mercado laboral se encuentra relacionada con el protagonismo asumido por los emprendimientos empresariales de mayor escala, que arribaron a la región en los últimos años, dirigidos de preferencia a la vitivinicultura. Dichas compañías, radicadas en lugares alejados de los centros urbanos poblados y orientadas en gran medida a la exportación de su producción, deben alcanzar certificaciones correspondientes a las denominadas buenas prácticas agrícolas, que repercuten de alguna manera en las formas de contratación y en las condiciones de trabajo.

Aunado a lo anterior se debe mencionar que aun cuando la aparición de tareas nuevas y la reformulación e importancia que adquieren otras realizadas a lo largo de la historia en la zona favorecerían una mayor estabilidad y continuidad en los ciclos laborales, éstos se caracterizan por una mayor eventualidad, debido a que se estructuran condicionados por las estrategias de los empresarios agrícolas tendientes a ajustar las contrataciones temporales al tiempo de trabajo utilizado de manera efectiva.

\footnotetext{
${ }^{5}$ Según el cNa de 2002, en el Valle de Uco existían 10900 hectáreas cultivadas con hortalizas, 15 mil con vid y 14500 con otros frutales.
} 
De esta forma, la contratación de empleo temporal se despliega en momentos diferentes del ciclo productivo por lapsos variables, en lugar de corresponder a un número reducido de tareas ubicadas en momentos definidos del año. Así, se suman a las clásicas tareas estacionales de podas de invierno y de cosecha, a las nuevas de poda en verde y de raleos que incorporan otros tiempos de contratación redefiniendo la temporalidad. Así se desdibuja su condición estacional y adquiere el mencionado tono de eventualidad.

Estas características de la demanda laboral dificultan más la compleja coordinación entre la demanda y la oferta laboral en producciones intensivas que requieren de mano de obra. La necesidad de movilizar grandes cantidades de fuerza de trabajo, para satisfacer los requerimientos por unidad de superficie y el tamaño de los establecimientos, constituye un problema tradicional que deben enfrentar este tipo de empresas, que se acentúa ante la eventualidad del empleo transitorio. De esta forma, no sólo hay que resolver la disponibilidad de mano de obra frente a la escasez de jornaleros locales, que por tradición se solucionaba empleando a los migrantes temporales movilizados a través de los clásicos enganchadores, sino que se debe solucionar una coordinación dificultosa entre un grupo de grandes establecimientos que demandan un gran contingente de personal por periodos breves y los trabajadores locales que lo conforman.

En ese sentido, la intermediación laboral se consolida como una institución que resuelve en lo cotidiano de manera flexible la demanda de trabajo temporal, cuyo perfil se ha tornado mucho más complejo. En esta situación, en la región sobresalen aspectos como la difusión de las modalidades de contratación indirecta de mano de obra transitoria en explotaciones de orientación productiva distinta a escala diferente, junto a su utilización en una gama mayor de tareas. De manera pormenorizada se puede observar que en los tres departamentos que conforman el Valle de Uco, 779 explotaciones agropecuarias (EAP) contratan mano de obra transitoria, mediante modalidades indirectas, para que se ocupe de 44192 hectáreas. $^{6}$

\footnotetext{
${ }^{6}$ Para el caso de los jornaleros transitorios contratados en forma indirecta, el cNA releva la superficie trabajada en hectáreas para la cual se contratan, y no mediante jornales como lo hace para los transitorios contratados de manera directa.
} 
Al igual que lo ocurrido con la contratación directa, es la intermediación de la contratación de tareas de cosecha la que está presente en la mayoría de estas explotaciones (85 por ciento), y explica la mayor parte de la superficie involucrada. Las labores de poda y las agrupadas en la categoría "otras formas de mantenimiento de cultivos" siguen en importancia a la cosecha tanto por el porcentaje de EAP que contratan, como para la superficie en la que se utiliza este tipo de trabajadores (véase figura 2).

En relación con las características de las empresas demandantes se puede observar que, inclusive en los terrenos inferiores a diez hectáreas, la intermediación laboral se difunde en tareas que exceden a la cosecha. Sin embargo, la importancia de las actividades para las cuales se contrata mano de obra de forma indirecta se manifiesta en mayor proporción en los campos de "mediana extensión". Para este tipo de contratación, la poda y raleo ofrecen mayores porcentajes de difusión a medida que aumenta el tamaño de las tierras, ya que la plantación y el mantenimiento de cultivo muestran comportamientos similares a lo largo de todas las escalas de extensión.

Los datos obtenidos del CNA muestran una difusión considerable de la intermediación y utilización de mano de obra transitoria. En este fenómeno sobresalen cada vez más las tareas realizadas antes de la cosecha, relacionadas con el mantenimiento y la calidad del cultivo, y un mayor peso de las unidades de mediana extensión en estos comportamientos laborales.

\section{La regulación institucional} de los mercados de trabajo agrícola en el Valle de Uco

En los escenarios nuevos, a la intermediación laboral le acompaña una diversidad de modalidades de contratación, no asociada en exclusiva al trabajo no registrado. Dicha variedad, utilizada para registrar a trabajadores, observa grados diferentes de cumplimiento de la legislación laboral. Por un lado, hay contratos que se ajustan a las exigencias legales y, por otro, a situaciones que implican formas de registro "sucias" o "parciales". Éstas incurren en incumplimientos 


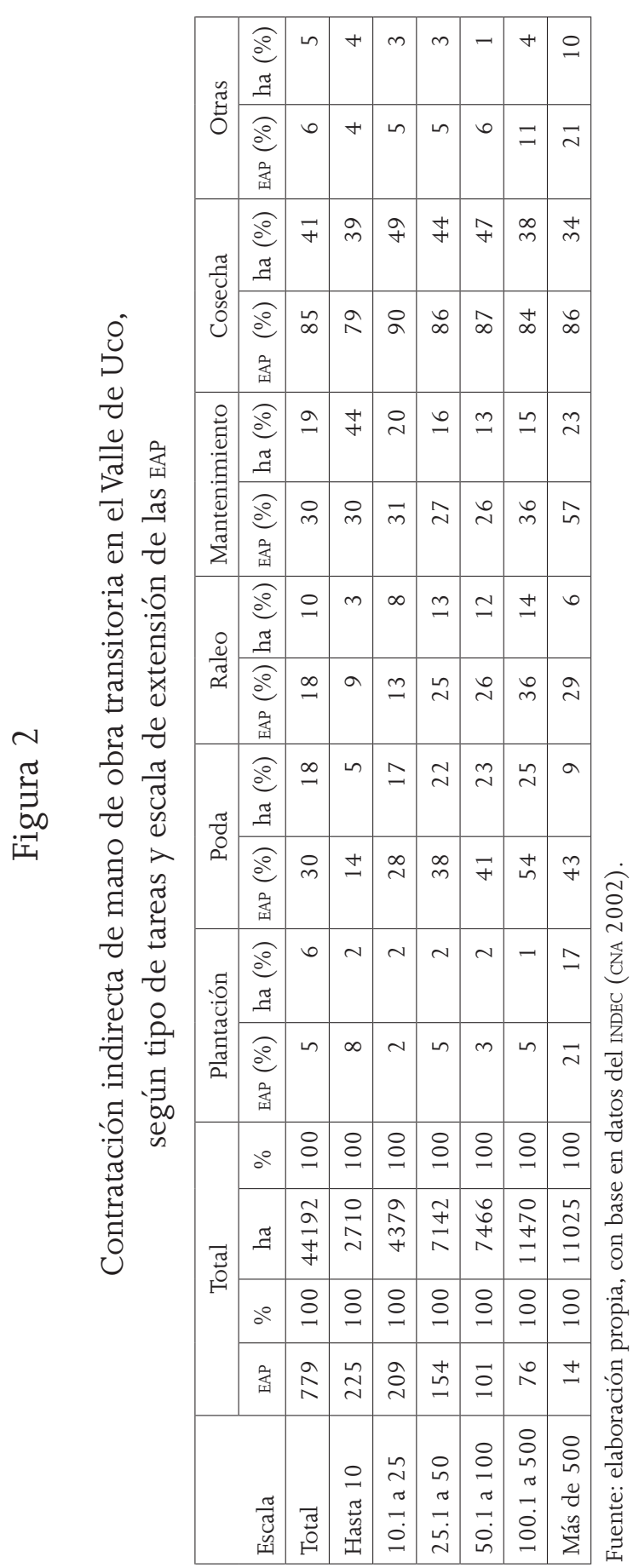


de la legislación o constituyen un fraude, es decir, disfrazan formas de trabajo dependiente y desdibujan la relación laboral.

Los regímenes y las modalidades de contratación en la agricultura del Valle de Uco se desprenden del Régimen Nacional del Trabajo Agrario (Ley 22.248), la Libreta del Trabajador Rural y el Registro Nacional de Trabajadores Rurales y Empleadores (RENATRE) (Ley 25.191) y de la Ley de Contrato de Trabajo y las leyes de reforma laboral $(20.744,25.013$ y 25.877). En el ámbito de influencia de la última legislación se ubica a quienes se dedican a actividades frutícolas, que se les excluye del régimen especial destinado al trabajo agrario. $^{7}$

Las alternativas de contratación, de que disponen los empresarios en el marco de las legislaciones vigentes, además de las modalidades de trabajadores permanentes o a plazo indeterminado (que no son centrales para el fenómeno en consideración) incluyen el contrato no permanente definido en Régimen Nacional de Trabajo Agrario y los de plazo fijo, de temporada y eventuales, contenidos en la Ley General de Contrato de Trabajo. Las dos últimas modalidades están difundidas en las actividades agrícolas encuadradas en la Ley General de Contrato de Trabajo y al margen del Régimen Nacional de Trabajo Agrario.

A los empleados contratados por las empresas de servicios eventuales se les considera permanentes discontinuos. Y éstas son responsables en primera instancia del cumplimiento de la legislación vigente, en tanto que, como es sabido, las firmas usuarias de la mano de obra son responsables solidarias de dicho acatamiento.

Las actividades encuadradas en el marco del Régimen Nacional de Trabajo Agrario tienen posibilidad limitada de contratar gente según la modalidad eventual de contrato. ${ }^{8}$ Frente a este escenario, las empresas principales de empleo eventual constituyeron otras de servicios de tareas agropecuarias en paralelo, para desempeñarse de forma temporal en estas tareas. De igual manera, debido a las mejores condiciones relativas de ocupación que brindan estas firmas, por

\footnotetext{
7 “Al trabajador ocupado en tareas de cosecha y/o empaque de frutas, el que se registra por el régimen de contrato de trabajo aprobado por la Ley No. 20744”.

${ }^{8}$ Según el artículo 2 de la resolución no. 14/2004 del RENATRE: "Disponer la no admisión de inscripciones en el RENATRE, de Empresas de Servicios Eventuales”.
} 
lo general los agentes encargados de la fiscalización destinan mayores esfuerzos a otro tipo de intermediarios, ubicados al margen de la legislación correspondiente.

Otra forma de contratación que tuvo una amplia difusión a principios de la década de 1990 fue la de las denominadas pseudocooperativas de trabajo, empresas de servicios de mano de obra camufladas en figuras asociativas que violan la legislación laboral registrando asalariados agrícolas como trabajadores autónomos falsos. Por consiguiente, a éstas se les ha tipificado como una modalidad de fraude, ya que son consideradas como meras intermediarias de la mano de obra dentro del mercado laboral, y así se han servido de una cierta apariencia cooperativa para sustraerse del cumplimiento de aportes y contribuciones. Es decir, se piensa que son compañías de prestación de servicios de mano de obra en actividades agrícolas disfrazadas como cooperativas (Bendini y Gallegos 2002; Tadeo et al. 2006).

En 1994 se prohibió la conformación de cooperativas de trabajo nuevas a través del decreto 2015/94. Después, el artículo 40 de la Ley 25.250 de 2004 estableció: "Las cooperativas de trabajo no podrán actuar como empresas de provisión de servicios eventuales, ni de temporada, ni de cualquier otro modo brindar servicios propios de agencias de colocación”. Sin embargo, y a pesar de la prohibición mencionada, algunas de ellas continúan operando, ya sea aduciendo su existencia previa a la normativa que restringe su actuación o desempeñándose en completa clandestinidad. En el primer escenario, éstas pueden presentarse en instancias judiciales para defender su derecho a funcionar en la actividad económica. ${ }^{9}$ Mientras que, en el segundo, despliegan estrategias para no ser vistas, y que no se les pueda ubicar para establecer su responsabilidad en caso de conflictos legales. Como práctica común, dichas cooperativas establecen un domicilio legal fuera de la provincia o uno inexistente, a la vez que cambian de denominación social ante las sanciones sucesivas. ${ }^{10}$

\footnotetext{
${ }^{9}$ Esta situación corresponde a la pseudocooperativa de trabajo principal del Valle de Uco, suspendida en fecha reciente debido a irregularidades diversas.

${ }^{10}$ Esta estrategia es común a la desplegada en otras provincias, donde a la vez se detectó un "mercado" de razones sociales de cooperativas de trabajo registradas antes de la prohi-
} 
Las cooperativas de trabajo y las empresas de empleo eventual compiten entre sí por las compañías demandantes, sus "clientes”, fundamentalmente a través del precio final del servicio prestado. Una forma de bajar costos o incrementar los beneficios consiste en registrar de manera parcial a su personal, y de la misma forma realizar los aportes respectivos. Esto puede lograrse, por ejemplo, cuando las cooperativas contratan sólo un número limitado de seguros de accidentes civiles, o si las empresas de contratación eventual declaran y aportan sólo por algunos de los días trabajados. Así, los actores involucrados externalizan las "negatividades", sus costos y obligaciones, transfiriéndolos al trabajador, es decir, el actor más débil y con menor poder.

La alternativa de flexibilizar en forma cuantitativa la contratación (posibilidad de tomar y desligarse de los empleados con las menores limitaciones posibles) no depende en exclusiva de la ausencia, escasez o falta de eficiencia de los controles ejercidos por los organismos correspondientes, ya que la variedad de modalidades de contratación disponibles facilita la flexibilización de la mano de obra, sin la necesidad de recurrir a formas típicas de trabajo no registrado. Así, los empresarios pueden acceder a una fuerza laboral precaria que, sin embargo, se puede encontrar registrada según lo establecido por la legislación. "Parece estar empezando a ocurrir sin embargo un curioso fenómeno que es el de convertir la precariedad en norma, o sea convertir en legales relaciones laborales antes consideradas< <negras>" (Murmis 1994, 53).

\section{Modalidades y funciones de la intermediación}

La subcontratación favorece las estrategias empresariales de gestión destinadas a establecer distancias y eslabones intermedios con los

\footnotetext{
bición mencionada. El valor de estas razones sociales dependía del estado que guardaba la documentación correspondiente presentada a las autoridades encargadas de supervisar este tipo de entidades. Dicho valor se incrementa a medida que resulta más sencilla la regulación de la documentación. También aconteció que frente a la prohibición y a la presión ejercida por la fiscalización, algunas de las cooperativas adquirieron la forma de empresa prestadora de servicios.
} 
trabajadores, evitar costos administrativos y problemas organizacionales, eludir las responsabilidades legales de la contratación y, en muchas ocasiones, llevar a la práctica formas que no respetan las normativas vigentes (Ortiz 2000). Inclusive, la utilización de muchos subcontratistas pequeños constituiría una barrera a los posibles procesos de organización gremial de los obreros, y restringiría las posibilidades de disputas a las resistencias y negociaciones en el lugar de trabajo (Ortiz y Aparicio 2006).

Así, la intermediación laboral desdibuja la relación de trabajo desplazando la tensión entre los empleados y los capitalistas hacia los intermediarios. También genera condiciones de asalarización, que reducen la capacidad de negociación del personal, favoreciendo la precarización de los mercados laborales (Aparicio et al. 2004; Alfaro 1999; Rau 2002).

En los últimos años, en la provincia de Mendoza la heterogeneidad de las modalidades y funciones de la intermediación laboral han ido en aumento. En los mercados de trabajo considerados, las figuras antiguas de contratistas o enganchadores de mano de obra, pseudocooperativas de trabajo, empresas de servicio y contratación eventual son las formas presentes y se combinan de modo diverso. En el Valle de Uco dicha heterogeneidad se visualiza en mayor medida debido a las modalidades y funciones que asume. A su vez, estas formas y funciones exhiben diferencias según el tipo de producción, las características de los establecimientos y sus estrategias organizacionales.

De este modo surge cierta asociación entre tipo de producción, de establecimiento o empresa y las modalidades de intermediación a las que se recurre. Por ejemplo, la producción de frutales en establecimientos pequeños y medianos, cuando se contrata trabajo temporal de forma indirecta, utiliza cuadrillas poco numerosas o intermediarios informales sin preocuparse, por lo común, del registro de los jornaleros. Cuando aumenta la dimensión de las empresas recurren, solas o combinadas, a alguna de las modalidades que permiten el registro de los trabajadores. Un escenario similar se presenta en la horticultura, pero las cooperativas aparecen a medida que aumenta la escala de los establecimientos, sobre todo cuando se encuentran articuladas con la etapa del empaque. Por su parte, 
en la vitivinicultura encuentra mayor grado de difusión, junto a las modalidades señaladas, es decir, las empresas de contratación eventual. En términos generales se puede indicar más registro de trabajadores en esta actividad, aunque no siempre su calidad es la óptima o indicada.

Entre las funciones que cumple aparecen algunas consideradas clásicas y otras que resultan más novedosas en el funcionamiento de estos mercados de trabajo. La movilización y el reclutamiento de la mano de obra, la organización y la supervisión, el alojamiento de los migrantes y la externalización de los aspectos formales de la relación laboral (registro de los trabajadores), se presentan de manera alternativa o combinada entre las funciones principales de la intermediación laboral. Así, ésta se puede construir recurriendo a uno o más de los agentes señalados, para cumplir alguna o todas las funciones indicadas.

La forma más simple de intermediación corresponde a la cuadrilla, integrada por su jefe o capataz y una composición cuyo tamaño es lo suficientemente flexible para adaptarse a varias tareas y momentos del año; suele ubicarse entre 15 y 30 personas, y es difícil que lleguen a 40 pues no podría ser supervisada por un solo jefe. Los cuadrilleros por lo general evidencian una trayectoria laboral en la cual las funciones de supervisor en una finca, de contratista de viña ${ }^{11}$ o de mediero en la horticultura representan los pasos previos para acceder al capital físico y social mínimo para desempeñar la tarea.

\footnotetext{
${ }^{11}$ Esta figura singular constituyó originalmente un tipo particular de trabajador y un actor clave en la expansión de la superficie implantada con vid. En una primera instancia, este actor denominado contratista de plantación — que residía en el establecimiento- se encargaba de implantar viñedos a cambio de una cantidad de dinero por cepa y de la producción obtenida en las primeras cosechas. Después, el contratista de viña estaba a cargo del riego, la poda, otras tareas de mantenimiento del cultivo, de la organización y supervisión de la cosecha y de la contratación del trabajo transitorio a cambio de un porcentaje de la producción. En un inicio esta figura constituyó un canal de capitalización y movilidad social para muchos inmigrantes que pudieron aprovechar esa coyuntura (Salvatore 1986; Richard Jorba 2003).

En fecha más reciente este actor pierde centralidad y se constituye en un trabajador permanente con un contrato atípico, con derecho a la seguridad social y jurídicamente diferenciado, cuyo sistema de remuneración combina una retribución mensual y un porcentaje de la cosecha que comercializa junto a su patrón.
} 
Las cuadrillas funcionan al margen de la normativa laboral vigente, y suelen estar integradas por pobladores locales; se encargan del reclutamiento y el transporte de los trabajadores, que se realiza en un vehículo propio o se contrata el servicio, también organizan y supervisan las tareas, y el jefe es el responsable por la disciplina y el desempeño laboral de los miembros de "su" cuadrilla. La remuneración del jefe se fija en función de la cantidad de jornaleros movilizados (un valor fijo por cada contratado) o a partir de un porcentaje de la labor realizada por "sus" trabajadores, y las magnitudes varían según las funciones asumidas o la distancia de las fincas. El origen social de estos capataces o jefes de cuadrilla es próximo al de los integrantes de ella, y los ingresos obtenidos por aquéllos en muchas ocasiones no resultan sustancialmente superiores.

Otra modalidad más compleja difundida en la zona corresponde a intermediarios que movilizan mayor cantidad de gente $y$, en consecuencia, utilizan más cuadrillas. Aquí, la figura del intermediario equivale a un prestador de servicios informal que - a lo sumo- se encuentra registrado de manera incorrecta según la modalidad de trabajador autónomo (monotributista). Estos intermediarios para reclutar mano de obra suelen recurrir a las cuadrillas ya formadas - como las descritas—, y a la contratación de jornaleros movilizados en forma individual.

La condición falsa de prestador autónomo de servicios, asumida por este tipo de intermediario, facilita la formalización parcial de la prestación del servicio al establecimiento, sólo desde el punto de vista impositivo. De igual forma, la contratación se mantiene al margen de la legislación vigente. Las funciones desplegadas por este agente y los contratos establecidos con los demandantes, mediante el que se abona el servicio, son similares a las indicadas para el caso anterior, aunque desde luego a mayor escala.

Los establecimientos que contratan personas a través de alguna o ambas de las figuras antes descritas y que, debido a sus dimensiones y notoriedad, se encuentran más expuestos a la fiscalización de los organismos correspondientes, o por las exigencias de los mercados con los que se articulan deben alcanzar cierta certificación, suelen requerir alguna forma de registro de los trabajadores. Para ello, es 
muy común que una vez contratado el personal recurran — según el tipo de registro buscado y los “costos" dispuestos a afrontar- a cooperativas de trabajo, empresas de contratación eventual o estudios contables que cuentan con compañías de servicio que sólo existen en "papel", para "anotar" a estos empleados. Agentes de este tipo suelen desplegar modalidades tendientes a reducir los "costos" mencionados, registrando a algunos de los trabajadores movilizados o manteniendo la posibilidad de que, por ejemplo, en caso de accidente se ajuste la nómina al momento de la liquidación de los jornales, e incluya al accidentado entre los formalizados.

Por otro lado, con respecto a los migrantes, se observa que los intermediarios siguen participando, según el caso y en diferente medida, en funciones tradicionales vinculadas al transporte, el alojamiento y en alguna financiación de los consumos al inicio de la temporada. Con respecto a la transportación, en la actualidad gran parte se realiza en autobuses y, en algunos casos, financiada a través de organismos públicos y sindicatos de la provincia de procedencia (por ejemplo, Tucumán). Inclusive, en los últimos años, empresas de contratación eventual con agencias en diferentes provincias comienzan a funcionar reclutando migrantes en sus lugares de origen.

El costo del pasaje de ida puede ser adelantado por el intermediario y después descontado de los ingresos del trabajador. En cambio, el de regreso puede ser una especie de bonificación para quienes permanecen ocupados con el mismo intermediario toda la temporada, que funciona como un aliciente para la retención de esa mano de obra. Para reforzar este dispositivo, puede suceder que se retenga un porcentaje del pago que se abona al final.

Otra forma de movilización la constituyen los programas de organismos gubernamentales y sindicatos. Por ejemplo, La Federación Obrera Tucumana de la Industria del Azúcar incluye a la provincia de Mendoza, entre los destinos más relevantes de los movilizados emtre las zafras (Giarracca et al. 2001). Los intermediarios pueden encargarse del alojamiento cuando no es en los establecimientos. En cuyo caso ellos poseen o tienen acceso a viviendas denominadas “colectivas", donde los jornaleros se alojan en condiciones muy deficitarias. 
Las cooperativas de trabajo conforman otra modalidad de intermediación muy difundida en el Valle de Uco. ${ }^{12}$ Algunas empezaron a operar antes de su prohibición y, como ya se mencionó basan su posibilidad de realizar actividades económicas en su preexistencia a dicha reglamentación. De manera paralela hay otras al margen de la normativa vigente. Un representante de una de ellas indicó que en la actualidad existen cinco de relevancia, que involucran a una proporción significativa de la mano de obra movilizada en la zona. La principal, según manifestó su responsable, contrata en los momentos de mayor demanda a alrededor de tres mil personas. Esta modalidad suma a las funciones ya señaladas el registro de estos trabajadores bajo la figura de asociados y, junto a ella, a modalidades específicas de economía social. Los aportes monetarios que se deben realizar por dichas figuras son sustancialmente inferiores a los que se deberían abonar en caso de no desdibujar la relación laboral bajo una forma asociativa falsa. Los aranceles que se cobran a las empresas por estos servicios varían, según las funciones desempeñadas, que se ubican en torno a 20 por ciento de los salarios percibidos por "sus" empleados.

Las dimensiones de estas "cooperativas" obligan a que las estructuras internas y formas de organización sean más complejas, sin embargo son muy flexibles, y están sostenidas por las relaciones personales de las figuras encargadas del reclutamiento y las posibilidades que brindan los medios de comunicación actuales para movilizar velozmente a los trabajadores, por ejemplo a través de la telefonía móvil.

La flexibilidad se manifiesta en la organización de la mano de obra. Cada "grupo de trabajo" — una cuadrilla — está a las órdenes de un "jefe de grupo", denominado así por las cooperativas, que corresponde al capataz o jefe de cuadrilla tradicional, y se encarga, junto a algún miembro de la empresa, de coordinar y supervisar

\footnotetext{
${ }^{12}$ A diferencia de lo acontecido en otras provincias y producciones, donde estas pseudocooperativas mutaron en empresas prestadoras de servicios con algún grado de formalización (Craviotti y Palacios 2007), no se observa en el presente caso un retroceso de esta figura de intermediación. Inclusive éstas continúan operando, a través de medidas de amparo judicial a pesar de las sanciones aplicadas por la autoridad correspondiente.
} 
la tarea por realizar. Cuando la cantidad de contratados involucra varios "grupos de trabajo" aparece la figura del "responsable"; y por último, por encima de éstos se encuentra el representante de la cooperativa en la zona.

Así, disponen de una red amplia de relaciones personales y no necesitan invertir en instalaciones físicas de cierta relevancia, por ejemplo oficinas. Estas compañías de servicios, camufladas como cooperativas, logran movilizar volúmenes muy significativos de jornaleros. En algunos casos, aprovechando las ventajas de este régimen, las cooperativas brindan el servicio de "registro" de los trabajadores. A través de esta modalidad logran algún grado de formalización del registro de la relación laboral a empresas que contratan a sus empleados de forma directa o mediante los agentes de intermediación ya analizados. Otra modalidad de gran importancia es la conformada por las firmas de servicios eventuales de mano de obra. En relación con dichos formatos se observa que, si bien existe un conjunto de ellas funcionando en la región según esta forma jurídica, hay una que se ha consolidado como el referente primordial del servicio y una de las empleadoras principales de la región, y está subdividida en tres empresas, las cuales ofrecen prestaciones como la mera administración del personal hasta servicios que incorporan la organización del trabajo, el transporte o la vivienda. De esta manera brinda una gama variada de posibilidades y precios a sus clientes, y a la vez se adapta al complejo entramado de marcos jurídicos del mercado de trabajo. A partir de la gran flexibilidad y conocimiento de los espacios jurídicos que el territorio permite desplegar, las empresas logran adaptar sus funciones, formatos y contratos a las normativas vigentes; situación que las diferencia de las cooperativas de trabajo, en lo que se refiere al tipo de formalización del vínculo laboral establecido con las personas que moviliza.

La posibilidad de contratar trabajo registrado sin establecer un nexo directo con la gente, y al mismo tiempo tener la oportunidad de ajustar esa contratación a la eventualidad de los requerimientos laborales, impulsa a los empresarios a utilizar la movilización de personal; y desligarse en la mayor medida posible del reclutamiento, la contratación y los aspectos legales, objetivos muy valorados por ellos. El costo del servicio ofrecido debe cubrir tanto los aportes 
laborales como el beneficio económico obtenido, cuya magnitud presenta el interrogante acerca de la difusión de la modalidad en una actividad que habitualmente opera en condiciones de trabajo no registrado. Las compañías expuestas en mayor medida a la fiscalización laboral, o las que deben alcanzar certificaciones para que sus productos lleguen a mercados internacionales pueden estar dispuestas a asumir esos valores mayores para contratar mano de obra según las exigencias legales, evitando al mismo tiempo vínculos directos con los trabajadores. También existe la posibilidad de reducir "costos", a través de los ardides referidos a los aportes por menos días a los realmente laborados.

La opción de registrar empleados sin establecer una relación directa generó una función nueva de la intermediación correspondiente al registro del vínculo laboral. Una forma de realizarlo consiste en la conformación, a través de estudios contables, de empresas de servicios que sólo existen en "papel” en las cuales se inscribe de manera formal a los trabajadores. Así, una compañía que utiliza cuadrillas pequeñas o enganchadores informales para contratar gente puede hacerlo mediante este servicio que intermedia el registro y terceriza las tareas administrativas, por ejemplo, de liquidación de salarios. El empleo de intermediarios se ha establecido a lo largo de la historia con el objetivo de atemperar posibles situaciones conflictivas, como las relativas a la disciplina, presentismo laboral, accidentes, interrupciones o montos de los jornales pagados.

La presencia de modalidades y funciones combinadas de la intermediación laboral genera una novedad en la naturaleza del fenómeno; además del reclutamiento y la contratación de la mano de obra, puede incluir su registro. Inclusive, en ocasiones incorporar funciones de coordinación de las tareas que exceden las clásicas de control y supervisión a cargo de los capataces o jefes de cuadrilla, en cuyos casos la intermediación laboral abarca procesos de tercerización de la organización productiva. ${ }^{13}$

\footnotetext{
${ }^{13}$ A veces la intermediación laboral sólo abarca el reclutamiento y la contratación de la mano de obra, mientras que en otras, comprende el desempeño de alguna tarea o etapa del proceso productivo, adquiriendo rasgos típicos de la denominada subcontratación laboral interna (Iranzo y Leite 2006).
} 
El papel de la intermediación y sus efectos en los mercados de trabajo

La difusión de la intermediación laboral influyó en las condiciones vigentes de los mercados de trabajo del agro en el Valle de Uco. La complejidad del fenómeno vinculado a la diversidad de formas asumidas y funciones desempeñadas altera el carácter que presenta la precariedad laboral, modificando su perfil y sus peculiaridades.

A lo largo de la historia, el trabajo agrario se ha distinguido por la estacionalidad, los salarios bajos, jornadas extensas y condiciones deficitarias, la poca sindicalización y la presencia generalizada de jornaleros no registrados. Debido a esta caracterización, las ocupaciones agrícolas se han identificado como flexibles y precarias (Piñeiro 1999).

Al mismo tiempo se reconoce que los nuevos escenarios presentes en las actividades agrarias profundizan la precariedad laboral y modifican su perfil. Así, en ésta, las condiciones vigentes coexisten con otros rasgos que resultan novedosos. Las características principales que asume la precariedad laboral en la agricultura son la intensificación de la eventualidad e intermitencia laboral, expresada en la multiocupación creciente de los asalariados agrícolas; el aumento de una oferta de trabajo cada vez más urbana y sólo asalariada; la emergencia de procesos de segmentación por género y etnia; la persistencia de sistemas de remuneración a destajo o por jornal, que asociados a la intermitencia laboral mencionada implican ingresos anuales bajos; la ausencia de contratos registrados y la preeminencia de arreglos verbales y las condiciones de trabajo y de vida degradantes, muchas veces agravadas por el carácter migrante de la persona (Lara Flores 2007; Piñeiro 2008).

La asociación entre las transformaciones de la intermediación laboral y los cambios en los perfiles de la precariedad en las ocupaciones agrarias del Valle de Uco puede analizarse considerando la estabilidad/inestabilidad de las ocupaciones, los ingresos obtenidos, la modalidad de contratación, el acceso a la seguridad social 
y las condiciones de trabajo, elementos que constituyen la noción clásica de este fenómeno. ${ }^{14}$

En relación con los ingresos se observa la persistencia de sistemas de remuneración a destajo, que mantienen inalterados los tradicionales salarios bajos vigentes en el sector. En algunas producciones y tareas pueden alcanzar percepciones diarias que, consideradas de forma aislada, resultan relativamente elevadas. Sin embargo, los ingresos anualizados ubican a los jornaleros y a sus familias en condiciones de marcada vulnerabilidad. ${ }^{15}$

En términos generales, en el Valle de Uco la intermediación laboral no perjudica mucho los salarios e ingresos, en comparación con los presentes en los contratos de trabajo establecidos de modo directo en el sector agrícola. Y en las condiciones laborales predominantes tampoco representan diferencias significativas según la modalidad de contratación. Las mejoras limitadas en cuanto a la precariedad ocupacional se vinculan a las regulaciones conocidas de forma genérica como buenas prácticas agrícolas, para acceder a mercados de exportación, y que establecen ciertas condiciones con respecto a normas de seguridad laboral, que repercuten en el Valle de Uco sobre todo en la producción de vides para la elaboración de vinos considerados de alta calidad enológica. Hay algunos avances, como la mejora en las condiciones de transporte, con la utilización de autobuses hacia los establecimientos agropecuarios, la presencia de baños químicos en los predios y, en menor medida, el alojamiento para los migrantes.

Sin embargo, la jornada de trabajo prolongada y su intensificación asociada a modalidades de pago a destajo y a laborar en situaciones expuestas a temperaturas altas y condiciones ergonómicas deficitarias, por ejemplo, reducen el efecto positivo de esas normas sobre las condiciones laborales vigentes en estas tareas.

${ }^{14}$ Aquí se abordan las características objetivas distintivas de las ocupaciones precarias. Diego Piñeiro (2008) avanza sobre la conceptualización del fenómeno e incluye rasgos subjetivos en la definición del trabajo precario en la agricultura.

${ }^{15}$ Ortiz y Aparicio (2007) profundizan acerca de la relación entre las ocupaciones en la actividad citrícola de exportación de la provincia de Tucumán y los límites que los ingresos discontinuos vigentes en ellas representan para las familias de dichos trabajadores, para ubicarse sobre la línea de pobreza. 
Así, mientras que las modalidades de intermediación laboral difundidas en el Valle de Uco no modifican de manera sustancial la precariedad de los ingresos y las condiciones de trabajo vigentes, su papel en los cambios experimentados por la temporalidad de las ocupaciones y su registro progresivo en modalidades distintas modifican el perfil de la precariedad laboral que por tradición ha caracterizado a la agricultura.

A diferencia de la estacionalidad productiva clásica de los ciclos de labor agrícola, donde se identifican marcados "picos" y "valles" en la demanda de trabajo, lo que se observa en el Valle de Uco es más bien la conformación de un mercado donde la estacionalidad en la demanda laboral se superpone a contrataciones eventuales para un número importante de tareas y jornaleros a lo largo del año. Esta situación responde a la incorporación de faenas nuevas y a la reformulación de algunas antiguas, vinculadas a la reconversión productiva en dirección a la calidad que atravesó la región.

Por otra parte, entre las funciones de la intermediación laboral surge el registro de las ocupaciones, que puede desempeñarse de tres formas: mediante empresas de contratación eventual o prestadoras de servicios, que realizan también otras tareas; a través de estudios contables que establecen en el "papel" firmas de servicios para anotar a los contratados por medio de intermediarios no formalizados o - en algunos casos- de manera directa y con pseudocooperativas de trabajo.

Las alternativas legales favorecen estrategias de contratación que posibilitan formas de registro diferentes de la mano de obra en el ramo agrícola. A la posibilidad de contratar trabajo eventual o no permanente, se suma el registro como socios de pseudocooperativas que - como es sabido- continúan operando amparadas en su preexistencia a la prohibición.

Al mismo tiempo, el registro de las ocupaciones según estas modalidades, en el mejor de los casos, permite el acceso parcial a la seguridad social. Inclusive, en aquéllas cuya inscripción se ajusta a lo establecido por la legislación, los jornaleros —debido a la inestabilidad de sus aportes- no alcanzan a disponer de una cobertura médica a lo largo de todo el año. Por su parte, en las ocupaciones establecidas a partir de pseudocooperativas, la cobertura médica se 
encuentra a cargo de mutuales que restringen y limitan los beneficios. Además, dicha modalidad utiliza seguros civiles, cuya protección es incierta e inferior a la brindada por las aseguradoras de riesgos del trabajo. Por último, la posibilidad de obtener una jubilación está condicionada por la inestabilidad de los aportes, derivada de la intermitencia laboral.

Las estrategias empresariales de registro mencionadas se centran en el "blanqueo" de las ocupaciones, con el propósito de evitar o reducir los "riesgos" de violar la legislación laboral. Algunas de estas formas de registro se contraponen a la legislación vigente, constituyendo modalidades caracterizadas como "sucias", a las cuales las empresas pueden recurrir gracias a los intersticios de la normativa. Incluso en el mejor de los escenarios, el registro de las ocupaciones no implica el "blanqueo" del total de los salarios, ya que las empresas pueden realizar aportes que cubran sólo algunos de los días trabajados, debido al pago por jornal, y generan situaciones catalogadas como "grises".

\section{A modo de conclusión: la intermediación laboral y el perfil de la precariedad laboral en el contexto de agriculturas reestructuradas}

La intermediación constituye un componente de la reestructuración agraria que articula flexibilidades, tanto de los procesos como de los mercados de trabajo, facilitando la contratación y el uso flexible de la mano de obra en el marco de estrategias empresariales orientadas a sostener los procesos de acumulación.

Asimismo, la intermediación adquiere una complejidad creciente, expresada en la multiplicidad de modalidades y en su diversidad de funciones. Los tradicionales enganchadores, las pseudocooperativas, empresas de contratación de trabajo eventual y los estudios contables, que registran de manera formal al personal contratado directa o indirectamente, están presentes en el fenómeno analizado. Estas formas pueden reclutar, transportar, alojar, supervisar, organizar o registrar a los empleados. Una particularidad destacada en los 
contextos actuales de reestructuración social y productiva corresponde a la articulación de modalidades y a la combinación de funciones a las que suelen recurrir las empresas que utilizan instancias de intermediación para la movilización de la fuerza de trabajo.

Esta forma de contratación, en un escenario caracterizado por una demanda laboral conformada por empresas de tamaños diversos, que requieren grandes contingentes de jornaleros en momentos distintos del año para el desempeño de tareas manuales, facilita el ajuste estricto de la contratación a los tiempos de trabajo efectivo. La preponderancia de la utilización de mano de obra local resulta clave para la conformación de esta eventualidad y la intermitencia laboral.

A su vez, la modalidad permite a las empresas desplegar estrategias de registro ocupacional intermediando el vínculo laboral en sus aspectos formales. Estas estrategias de registro son factibles gracias a los intersticios de la legislación y a las dificultades y carencias que enfrenta la fiscalización. La formalización de las ocupaciones es acompañada por la intermitencia laboral, los ingresos bajos y las condiciones de trabajo deficitarias. Así, la intermediación laboral en el Valle de Uco redefine la precariedad ocupacional en la agricultura, adquiriendo un perfil eventual y registrado.

Recibido en agosto de 2009 Aceptado en junio de 2010

\section{Bibliografía}

Alfaro, María Inés. 2003. Estrategias empresarias: exportación, intermediación y precarización en la citricultura tucumana. Ponencia presentada en el $6^{\circ}$ Congreso nacional de estudios del trabajo: los trabajadores y el trabajo en la crisis, Buenos Aires.

1999. Los espacios para la negociación laboral en la citricultura tucumana: actores y estrategias. Disciplinamiento, conflictividad y resistencias. Estudios del Trabajo 18: 39-59. 
Aparicio, Susana. 2005. Trabajos y trabajadores en el sector agropecuario de la Argentina. En El campo argentino en la encrucijada. Estrategias y resistencias sociales, ecos en la ciudad, coordinado por Norma Giarracca y Miguel Teubal. Buenos Aires: Alianza Editorial.

, P. Berenguer y V. Rau. 2004. Modalidades de intermediación en los mercados de trabajo rurales en Argentina. Cuadernos de Desarrollo Rural 53: 59-79.

Bendini, Mónica y Norma Gallegos. 2002. Precarización de las relaciones laborales y nuevas formas de intermediación en un mercado tradicional de trabajo agrario. Políticas Agrícolas 12: 46-67.

Bisio, Raúl y Floreal Forni. 1976. Economía de enclave y satelización de mercados de trabajo rural. El caso de los trabajadores con empleo precario en un ingenio azucarero del noroeste argentino. Desarrollo Económico 16 (61): 3-56.

Bocco, Adriana, Clara Martín y María Pannunzio. 1999. Mendoza: agricultura y ruralidad. Reflejos en la estructura social y agraria del oasis rural de San Carlos, Mendoza. Mendoza: Universidad Nacional de Cuyo, Editorial de la Universidad Nacional de Cuyo.

Brass, Tom. 2004. Medieval Working Practices. British Agriculture and the Return of the Gangmaster. Journal of Peasant Sudies xxxI (2): 313-340.

Castellanos Ortega, M., y A. Pedreño Cánovas. 2001. Desde el ejido al accidente de Lorca. Las amargas cosechas de los trabajadores inmigrantes en los milagrosos vergeles de la agricultura mediterránea. Sociología del Trabajo 42: 3-31.

Craviotti, C., C. Cattaneo y P. Palacios. 2008. La conformación del mercado de trabajo estacional vinculado al arándano en Entre Ríos, Argentina: estrategias empresariales y mecanismos de regulación social. Revista Latinoamericana de Estudios del Trabajo 13 (19): 173-94. 
2007. La trama detrás de la escena: los contratistas de servicios de mano de obra en la producción de frutas frescas de alto valor. Estudios del Trabajo 33: 3-28.

Fabio, José Francisco. 2006. El trabajo estacional en la agricultura. Tipos de trabajadores y estrategias laborales en la provincia de Mendoza, ponencia presentada en el viI Congreso latinoamericano de sociología rural, Quito.

Fisher, Lloyd. 1964. The Harvest Labor Market in California. Quarterly Journal of Economics LXv: 463-491.

Giarracca, N., K. Bidaseca y D. Mariotti. 2001. Trabajo, migraciones e identidad en tránsito: los zafreros en la actividad cañera tucumana. En ¿Una nueva ruralidad en América Latina?, compilado por Norma Giarracca. Buenos Aires: Consejo Latinoamericano de Ciencias Sociales.

Guibourdenche de Cabezas, Marta. 1976. Migración boliviana en Mendoza. Mendoza. Mimeografiado, Conicet, Universidad de Cuyo.

Herrán, Carlos. 1979. Migraciones temporarias y articulación social: el Valle de Santa María, Catamarca. Desarrollo Económico 19 (74): 161-187.

INDEC. 2002. Censo nacional agropecuario. Buenos Aires.

2001. Censo nacional de población, hogares y vivienda. Buenos Aires.

Iranzo, C., y M. Leite. 2006. La subcontratación laboral en América Latina. En Teorías sociales y estudios del trabajo: nuevos enfoques, coordinado por Enrique de la Garza Toledo. Barcelona: Anthropos Editorial.

Lara Flores, Sara. 2007. La precarización del trabajo agrícola en zonas de agricultura moderna-empresarial en México. XXviI International Congress of the Latin American Studies Asociation (LASA), Montreal. 
1998. Nuevas experiencias productivas y nuevas formas de organización flexible del trabajo en la agricultura mexicana. México: Juan Pablos Editor.

Marañón, Boris. 2000. Contratistas en mercados hortícolas de exportación en México: funciones económicas. Cuadernos Agrarios 19: 215-233.

Marx, Karl. 1975. El capital, tomo I, volumen 3. Madrid: Siglo XxI.

Murmis, Miguel. 1994. Algunos temas para la discusión de la sociología rural latinoamericana: reestructuración, desestructuración y problemas de incluidos excluidos. Ruralia 5: 43-68.

Neiman, Guillermo. 2003. La 'calidad' como articulador de un nuevo espacio productivo y de organización del trabajo en la vitivinicultura mendocina. En El campo en la sociología actual. Una perspectiva latinoamericana, compilado por M. Bendini, S. Cavalcanti, M. Murmis y P.Tsakoumagkos. Buenos Aires: Editorial La Colmena.

A. Bocco y C Martín. 2001. Tradicional y moderno. Una aproximación a los cambios cuantitativos y cualitativos de la demanda de mano de obra en el cultivo de vid. En Trabajo de campo. Producción, tecnología y empleo en el medio rural, compilado por Guillermo Neiman. Buenos Aires: Ediciones Centro de Integración, Comunicación, Cultura y Sociedad.

y Germán Quaranta. 2000. Reestructuración de la producción y flexibilidad funcional del trabajo agrícola en la Argentina. Revista Latinoamericana de Estudios del Trabajo 6 (12): 45-69.

Ortiz, Sutti. 2000. La reestructuración de la industria agrícola y los contratos laborales. Revista Latinoamericana de Estudios del Trabajo 6 (12): 29-44.

y S. Aparicio. 2007. How Labourers Fare in Fresh Fruit Export Industries: Lemon Production in Northern Argentina. Journal of Agrarian Change 7 (3): 382-404. 
2006. Contracts, Control and Contestation: The Harvest of Lemons for Export. The Journal of Peasant Studies 33 (2): 161-188.

Pedreño Cánovas, Andrés. 1999. Del jornalero agrícola al obrero de las factorías vegetales. Madrid: Ministerio de Agricultura, Pesca y Alimentación.

Piñeiro, Diego. 2008. El trabajo precario en el campo uruguayo. Montevideo: Comisión Sectorial de Investigación Científica, Facultad de Ciencias Sociales, Universidad de la República.

1999. Trabajadores rurales y flexibilidad laboral. El caso de Uruguay. En Empelo rural en tiempos de flexibilidad, coordinado por S. Aparicio y R. Benencia. Buenos Aires: Editorial La Colmena.

Radonich, Martha, Norma Steimbreger y Analia Kreiter. 2007. Reestructuración productiva en regiones frutícolas de exportación. Marco teórico metodológico para el estudio de estrategias empresariales. En Reestructuraciones sociales en cadenas agroalimentarias, compilado por Martha Radonich y Norma Steimbreger. Buenos Aires: Cuadernos del Gesa 6, Editorial La Colmena.

Rau, Víctor. 2002. Condiciones para la venta de capacidad laboral cosechera en el mercado de trabajo de la yerba mate. Estudios del Trabajo 24: 103-115.

Reboratti, Carlos. 1974. Santa Victoria. Estudio de un caso de aislamiento geográfico. Desarrollo Económico 55: 481-505.

y C. Sabalain. 1981. Vendimia, zafra y alzada: migraciones estacionales en la Argentina. Buenos Aires: Centro de Estudios de Población.

Richard Jorba, Rodolfo. 2003. El mercado de trabajo vitivinícola en la provincia de Mendoza y los nuevos actores. El contratista de viña: aproximación a un complejo sistema de empresarios y trabajadores. Revista Interdisciplinaria de Estudios Agrarios 18: 5-37. 
Rutledge, Ian. 1987. Cambio agrario e integración. El desarrollo del capitalismo en Jujuy: 1550-1960. Antropología Social e Historia. Serie Monográfica. Buenos Aires: Centro de Investigaciones en Ciencias Sociales.

Salvatore, Ricardo.1986. Control de trabajo y discriminación: el sistema de contratistas en Mendoza, Argentina, 1880-1920. Desarrollo Económico 26 (102): 229-253.

Sánchez Saldaña, Kim. 2006. Los capitanes de Tenextepango. Un estudio sobre la intermediación cultural. México: Miguel Ángel Porrúa Librero-Editor.

Tadeo, Nidia, Paula Palacios y Fernanda Torres. 2006. Agroindustria y empleo. El complejo citrícola del noreste entrerriano. Buenos Aires: Editorial La Colmena.

Whiteford, Scott. 1981. Workers form the North. Plantations, Bolivian Labor and the City in Northwest Argentina. Austin: University of Texas Press. 
